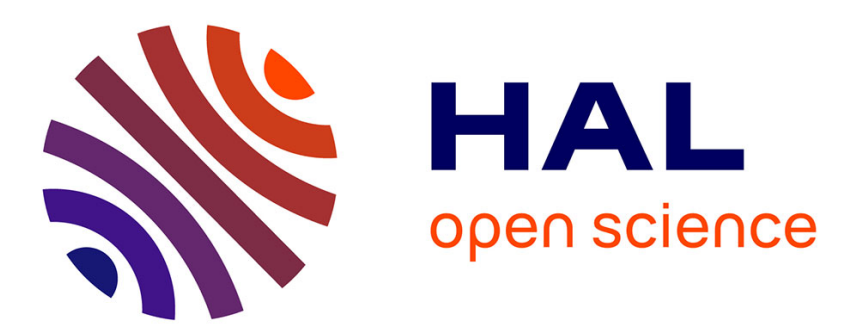

\title{
Joint 3D alignment-reconstruction multi-scale approach for cryo electron tomography
}

Hmida Rojbani, Etienne Baudrier, Benoît Naegel, Loïc Mazo, Atef Hamouda

\section{To cite this version:}

Hmida Rojbani, Etienne Baudrier, Benoît Naegel, Loïc Mazo, Atef Hamouda. Joint 3D alignmentreconstruction multi-scale approach for cryo electron tomography. IEEE International Symposium on Biomedical Imaging (ISBI 2016), Apr 2016, Prague, Czech Republic. hal-01318729

\section{HAL Id: hal-01318729 \\ https://hal.science/hal-01318729}

Submitted on 19 May 2016

HAL is a multi-disciplinary open access archive for the deposit and dissemination of scientific research documents, whether they are published or not. The documents may come from teaching and research institutions in France or abroad, or from public or private research centers.
L'archive ouverte pluridisciplinaire HAL, est destinée au dépôt et à la diffusion de documents scientifiques de niveau recherche, publiés ou non, émanant des établissements d'enseignement et de recherche français ou étrangers, des laboratoires publics ou privés. 


\title{
JOINT 3D ALIGNMENT-RECONSTRUCTION MULTI-SCALE APPROACH FOR CRYO ELECTRON TOMOGRAPHY
}

\author{
Hmida Rojbani ${ }^{\star \dagger} \quad$ Étienne Baudrier Benô̂t Naegel $^{\star} \quad$ Loüc Mazo $^{\star} \quad$ Atef Hamouda \\ * University of Strasbourg, CNRS ICube, ILLKIRCH, FRANCE \\ ${ }^{\dagger}$ University of Tunis ElManar, LIPAH, TUNIS, TUNISIA \\ Email: $\{$ hrojbani baudrier loic.mazo b.naegel\}@unistra.fr atef_hammouda@yahoo.fr
}

\begin{abstract}
$3 \mathrm{D}$ volume reconstruction in cryo-electron tomography is possible by using Transmission Electron Microscope (TEM) images from different tilt angles. The misalignment of these images is one of the limits to the quality of the reconstructed object. There are many alignment techniques to deal with this problem. Their common feature is to correct the 2D geometric transformation in the projection images. Nevertheless, 3D geometric transformation can occur in the TEM acquisition including tilt angular uncertainty. In this paper, we proposed a new multi-scale approach based on a Conjugate Gradient optimization of a cost function between the 3D reconstructed and the projection images with the purpose to find all the 3D parameters of geometric transformation. Tests on synthetic and real data prove the accuracy of our geometric transformation estimation.
\end{abstract}

Index Terms - Electron tomography, 3D reconstruction, Geometric transformation uncertainty, Conjugate Gradient, Multi-scale.

\section{INTRODUCTION}

Cryo-electron tomography (Cryo-ET) provides a way to investigate the inner structure of the biological cell in its natural state. The idea is to reconstruct a 3D model of the specimen structure using a recorded series of $2 \mathrm{D}$ projections captured by tilting the specimen under the electron beam. Different schemes of tilting around an axis normal to the electron beam can be used ( $c f$. Fig.1), but usually the maximum range of tilting is $\pm 70^{\circ}[1]$.

Numerous reconstruction methods are used in cryo-ET, mainly belonging to two families. First, the analytic family, with methods as filtered back-projection or direct Fourier inversion, implemented in Fourier's space [2]. The second one is the algebraic family, with iterative real-space methods such as ART [3] or SIRT [4].

There are several factors that can reduce the quality of the reconstruction such as: $(i)$ the low electron dose used during the acquisition to minimize the weight of radiation damage [5]; (ii) the thickness of the ice used in the sample fixation

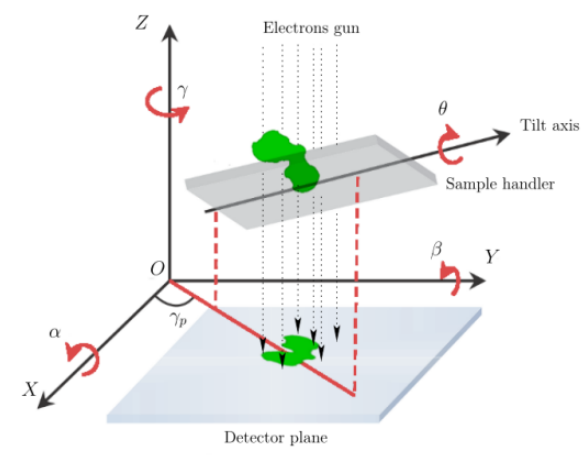

Fig. 1. TEM acquisition mechanism.

[6]; (iii) the missing data corresponding to the uncovered projection space; (iv) the uncertainty on the angular information due to mechanical imprecision of the microscope [7]; (v) the error made on the projection geometric transformation. The electron dose, the ice thickness and the missing data are an acquisition problem that cannot be corrected directly. Their effect on the reconstructed results are minimized in a postprocessing step. The angular uncertainty is considered as a negligible factor [7], which leaves it untreated by the majority of the proposed alignment approach to correct the projection geometric transformation. In fact, the resolution of the reconstruction at the time when [7] was published was mediocre, so the angular uncertainly does not have the effect over it. Nowadays, and due to the improvement of the reconstruction techniques, the resolution becomes important especially for the reconstruction and the location small particles such as ribosomes and nucleosomes. Treating the angular uncertainty will permit a better reconstruction resolution.

The two well common alignment techniques are [8]: (1) marker tracking, where gold particles are implanted into the samples before the image acquisition, then these markers are used to align the images [9], [10]; (2) pattern matching with cross-correlation methods, where alignment is carried out by cross-correlation calculation between each successive couple of images [11], [12]. 
In our work, we focus on the marker free methods. In fact, two successive projection images are not identical but similar and we can use them to detect the alignment parameters. A deficiency of this technique is the accumulation of errors from one alignment pair to another [13]. In addition, a stretching according to the cosine of the tilt angle for each projection image is needed before the alignment to establish a common area between the images for the technique to work [14].

To overcome those weakness, Dengler proposed in [15] a multi-resolution approach to refine the alignment parameters by coupling each projection image with its respective re-projection image from the reconstructed object. Many development of this method were proposed. The most recent one is the method of Tran et al. [16]. They proposed a hierarchical method to correct the reconstruction and the alignment problems in an alternating way. They treat the transformation parameters (translation, rotation, scale). It is inspired from the cross-correlation method, beginning by finding the first set of transformation parameters by an optimization between each successive pair of projection images. After that, a first reconstruction is applied, followed by a refinement of the set of transformations. If the method has not yet converged, they repeat the reconstruction with the founded set of transformations.

The defect of all these proposed methods is the fact that they only correct the alignment of the $2 \mathrm{D}$ projection images, which cannot cover all the possible 3D geometric transformation that can occur in the acquisition process. For that reason, we propose a new approach targeting the correction of the $3 \mathrm{D}$ geometric transformation on the reconstructed object directly. The background and our method are introduced in Section 2 , then our method is tested in Section 3 and a conclusion is given in Section 4.

\section{JOINT 3D ALIGNMENT-RECONSTRUCTION MULTI-SCALE APPROACH}

Correcting directly the $3 \mathrm{D}$ reconstructed object will be more accurate than correcting first the 2D projection images with each other, and then reconstructing the 3D object. Especially for the cryo-ET, where the missing wedge problem affect the performance of the reconstruction method. Our idea is to seek the 3D transformation parameters for each tilt angle that produces a projection image similar to its corresponding real one and simultaneously to correct the reconstructed 3D object. We use a hierarchal multi-scale image decomposition process ( $c f$. Fig.2), to estimate the transformation parameters in a coarse to fine way. In [17], we presented our first attempt to correcting the angle uncertainty. Here, we present our full approach of 3D alignment-reconstruction. We first present the used algebraic model.

Let $\mathbf{f}=\left(\mathrm{f}_{1}, \mathrm{f}_{2}, \ldots, \mathrm{f}_{\mathbf{N}}\right)^{T}$ be the vector including the $N_{1} \times$ $N_{2} \times N_{3}$ image $f$ to be reconstructed, where $\mathbf{N}=\Pi_{d=1}^{3} N_{d}$ is the number of voxels of $f$. Let $p_{i}$ be the $2 \mathrm{D}$ projection

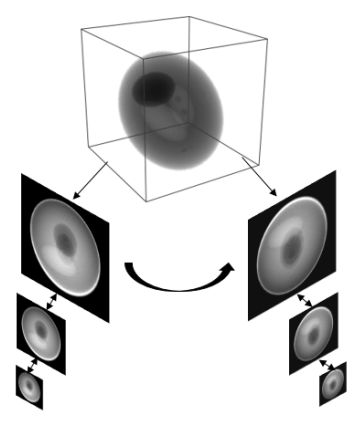

Fig. 2. Multi-scaling the original projections to iteratively correct the transformation parameters and improve the 3D reconstructed model.

image of size $M=M_{1} \times M_{2}$ according to the $\mathrm{i}^{\text {th }}$ tilt angle. Then, the vector form of $p_{i}$ is $\mathbf{p}^{i}=\left(\mathrm{p}_{1}^{i}, \ldots, \mathrm{p}_{\mathbf{M}}^{i}\right)^{T}$. We note $\mathbf{S}$ the number of acquired projection images and $\mathbf{P}=\left(\mathbf{p}^{1}, \ldots, \mathbf{p}^{i}, \ldots, \mathbf{p}^{\mathbf{S}}\right)$.

Let us talk now about the possible geometric transformation undergone by the 3D image $f$ during the acquisition. We present first the translation model, then the rotation one.

Suppose that the detector plan is parallel to the $(O X, O Y)$ plan. The image can translate in $(O X, O Y)$, which leads to the same translation over the detector plan. The translation of the image according to $(O Z)$, which is the focal axis, will produce scaling effect in the projection image. Thus, as we begin our approach by using a 3D estimated object, the 3D movement of this object can describe directly, at the same time, the 2D translation and the 2D scaling in the projection image $p_{i}$. These transformations will disturb the discretization of the reconstruction space. For that raison, we use the interpolation by means of B splines to prevent this problem. Let $\mathrm{T}$ be a $\mathbf{N} \times \mathbf{N}$ translation matrix applied on the vector $\mathbf{f}$ :

$$
\mathbf{f}^{i}=\mathrm{T}\left(t x_{i}, t y_{i}, t z_{i}\right) \mathbf{f}
$$

Each line of $\mathrm{T}$ describes the equation of translation with the interpolation according to $\left(t x_{i}, t y_{i}, t z_{i}\right)$, where the $\mathrm{k}^{t h}$ line of $T$ contains the interpolation coefficients to find the translated voxel $\mathrm{f}_{k}^{i}$.

We consider now the rotation. Usually, there are two types of rotation in cryo-ET, $(i)$ the rotation generated by the tilt angle and $(i i)$ the rotation that may occur in the detector plan. Using the Euler angular system $(\alpha, \beta, \gamma)$, we can present both rotations in the same time. We will use the same approach we used for the translation above. In our work, we do not apply the rotation on the image, but instead, we apply it to the projection lines. In fact, we use a $\mathbf{M} \times \mathbf{N}$ matrix $W$ which project the vector $\mathbf{f}$ into the vector $\mathbf{p}^{i}$ according to the angles $\left(\alpha_{i}, \beta_{i}, \gamma_{i}\right)$ as:

$$
\mathbf{p}^{i}=\mathrm{W}\left(\alpha_{\mathrm{i}}, \beta_{\mathrm{i}}, \gamma_{\mathrm{i}}\right) \mathbf{f}
$$

In fact, each line of the matrix $W$ describes one of the projection lines passing throws the image. Many methods are proposed to calculate this matrix such as "voxel driven" and "ray 
driven" [18]. However, in our work, the calculation of W is based on the "distance driven" method proposed by [19]. One must mention that both matrices $\mathrm{T}$ and $\mathrm{W}$ are sparse.

Our aim in this work is to correct every possible geometric error that can take place in the reconstruction step. The input data is a set of uncertain tilt angles and the corresponding real projection images. Hence, we propose to use a function that measures the distance between the real projection data and the projection data found after re-projecting the reconstructed volume according to the each current triple of angles $\left(\alpha_{i}, \beta_{i}, \gamma_{i}\right)$ and its translation $\left(t x_{i}, t y_{i}, t z_{i}\right)$. Thus, by refining all these parameters, we can assure having a more accurate reconstructed object.

We begin by defining this cost function $\mathcal{C}$ which stands for the Euclidean distance between the real set of projection data $\Pi$ and the re-projection data from the reconstructed object $\mathbf{P}$, so we have:

$$
\begin{aligned}
\mathcal{C}(\mathbf{f}, \boldsymbol{\alpha}, \boldsymbol{\beta}, \boldsymbol{\gamma}, \mathbf{t x}, \mathbf{t y}, \mathbf{t z}) & =\frac{1}{2}\|\mathbf{\Pi}-\mathbf{P}\|_{2}^{2} \\
& =\frac{1}{2} \sum_{i=1}^{\mathbf{S}}\left\|\boldsymbol{\pi}^{i}-\mathbf{p}^{i}\right\|_{2}^{2}
\end{aligned}
$$

This equation can be developed as:

$$
\mathcal{C}(\mathbf{f}, \ldots, \mathbf{t z})=\frac{1}{2} \sum_{i=1}^{\mathbf{S}} \sum_{j=1}^{\mathbf{M}}\left(\pi_{j}^{i}-\mathrm{p}_{j}^{i}\right)^{2}
$$

where,

$$
\begin{aligned}
\mathrm{p}_{j}^{i} & =\sum_{k=1}^{\mathbf{N}} w_{j k}\left(\alpha_{i}, \beta_{i}, \gamma_{i}\right) \mathrm{f}_{k}^{i} \\
& =\sum_{k=1}^{\mathbf{N}} w_{j k}\left(\alpha_{i}, \beta_{i}, \gamma_{i}\right)\left(\sum_{l=1}^{\mathbf{N}} t_{k l}\left(t x_{i}, t y_{i}, t z_{i}\right) \mathrm{f}_{l}\right)
\end{aligned}
$$

The cost function $\mathcal{C}$ depends on four parameters, the current projections, the current reconstructed volume, the current triple of tilt angles and the set of translation parameters for each triple of angles.

We use the square in the equation (3) to ensure the positivity of the function. The similarity between the two types of projections reaches its maximum when the function $\mathcal{C}$ reaches its minimum. In this case, the solution set of our problem is:

$$
(\widehat{\mathbf{f}}, \widehat{\boldsymbol{\alpha}}, \widehat{\boldsymbol{\beta}}, \widehat{\boldsymbol{\gamma}}, \widehat{\mathbf{t x}}, \widehat{\mathbf{t y}}, \widehat{\mathbf{t z}})=\operatorname{argmin} \mathcal{C}(\mathbf{f}, \ldots, \mathbf{t z})
$$

The equation (8) is approximately convex in $\mathbf{f}$ (due to the using of a quadratic sum) and the set of angles and translation parameters are close to the solution by hypothesis. We can therefore assume that in this case, the optimization problem of Eq. (8) is convex. Moreover, it is plain that the cost function $\mathcal{C}$ is not linear according to its rotation parameters. Thus, we have to use a non-linear minimization algorithm. Among the known deterministic optimization algorithms mentioned in the literature [20], we choose the non-linear version of the Conjugate Gradient (CG) algorithm [21]. The CG method is the most prominent iterative method for solving sparse systems of equations. We preferred this algorithm for its convergence rate, its simple implementation and since it appeals only to the first order derivative.

The first step to use the CG is to calculate the gradient of our cost function $\mathcal{C}$. In order to facilitate this phase, we have switched the equation (5) as:

$$
\mathcal{C}(\mathbf{f}, \ldots)=\frac{1}{2} \sum_{i=1}^{\mathbf{S}} \sum_{j=1}^{\mathbf{M}}\left(\mathcal{C} r\left(\mathbf{f}, \alpha_{i}, \beta_{i}, \gamma_{i}, t x_{i}, t y_{i}, t z_{i}, j\right)\right)^{2}
$$

with $\mathcal{C} r(\mathbf{f}, \ldots, j)=\pi_{j}^{i}-\sum_{k=1}^{\mathbf{N}} w_{j k}\left(\alpha_{i}, \beta_{i}, \gamma_{i}\right) \mathrm{f}_{k}^{i}$.

We first compute $\nabla \mathcal{C} r$, then $\nabla \mathcal{C}$. Due to the discrete nature of the cost function, we use the finite difference to estimate the gradient values. We use the Meyer wavelet to establish the multi-scale hierarchal decomposition [22].

After this decomposition, the roughest scale of the projection set is used with the given tilt angles to initialize our process. Then alternatively, the tilt angles, transform parameters and the reconstructed volume are optimized and the resolution is refined. Alg.1 summarizes our method.

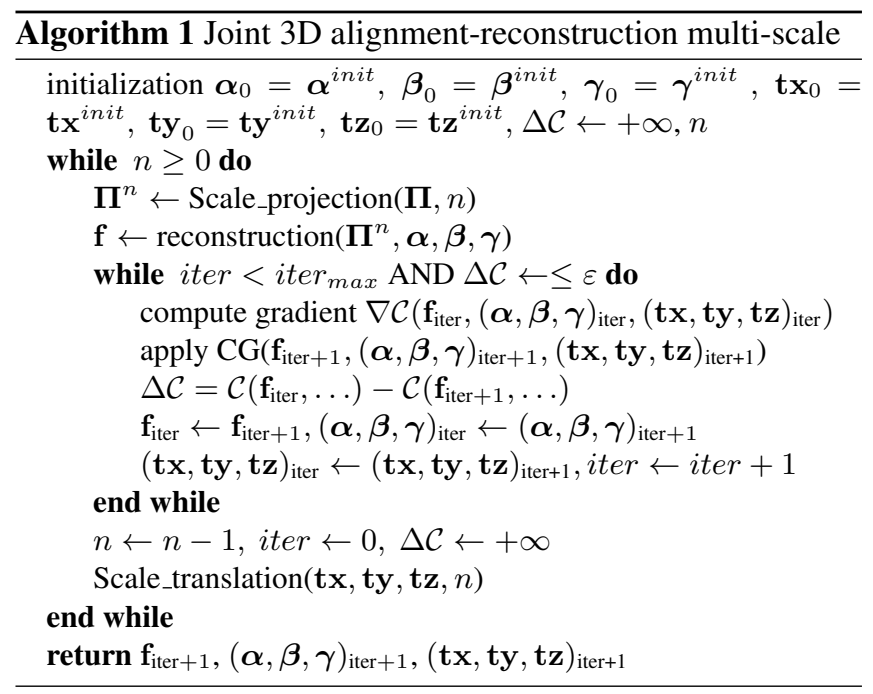

\section{EVALUATION OF THE METHOD}

We present in this section the experimental results of the proposed approach and a reconstruction done by using SIRT with the alignment method of projections given by [11].

Experiments are conducted on 25 gray-level 3D images of size $128^{3}$. An example of this base is given in Fig.3.

For each image, a set of projections uniformly distributed is computed using a set of tilt angles in $\left[-70^{\circ} 70^{\circ}\right]$. A random error of translation and rotation is been assigned to each projection image. In addition, a random error is assigned to the 
used tilt angles. In these experiments, we start the process by decreasing the scale twice. We find in these tests that the execution time is around $8 \times$ faster when we decrease by each scale. Due to the lack of place in this paper, we only present the final results. For each of the 25 images, 6 reconstructions are carried out and only the one with the minimum cost value is retained. In fact, the 6 reconstructions are slightly different, due to the estimation process of the $\alpha$ slop in the CG, which is using a fixed number of iterations.
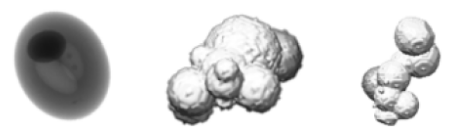

Fig. 3. Samples of 3D images from the synthetic base.

To evaluate the results, we compute the Mean Square Error (MSE) between the real data and i) the reconstructed images by our method ii) the direct reconstruction after an optimized alignment by the method proposed in [11].

$$
M S E(\%)=\frac{1}{\mathbf{N}} \sum_{i=1}^{\mathbf{N}}\left(\mathrm{f}_{i}-\widehat{\mathrm{f}}_{i}\right)^{2}
$$

Fig.4 shows the results for the 25 images. For all the tests, the MSE is between [0.10 0.35] for our approach, and between $[0.400 .85]$ for the other one. Our approach gives the best results for all the cases, due to the correction that includes not only the error on the geometric transformation but also the error affecting the tilt angles.

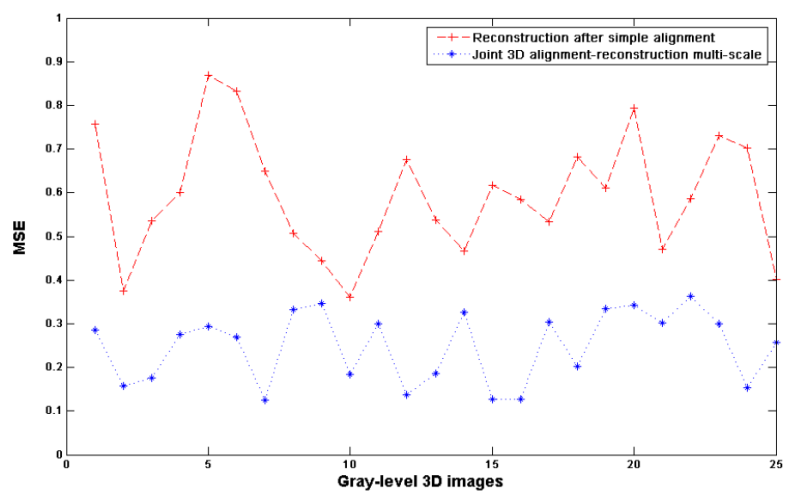

Fig. 4. Evaluation of the reconstruction performance using our approach and using the 2D alignment.

The proposed method is also applied to real data, which are projections of the virus of the Orf disease. Fig.5 presents some of the projections of the Orf-parapoxvirus. A reconstructed volume from the given projections and the given tilt angles with IMAGIC software [23], then filtered by experts, is also provided. The results are shown in Fig.6. The MSE between the real projections and the re-projections from our reconstructed object is equal to 0.36, where the MSE between the real projections and the re-projections from the reference volume is equal to 0.53 . A reason for this better result is that the angles have been modified by $0.0847^{\circ}$ in average by our process. Thus, our method was able to reconstruct some part that the other method failed to reconstruct. Another reason is the filtering of the reference volume that improves the volume rendering but reduces the matching to the data. The step was not applied to our reconstruction to emphasize the angle modification effect.

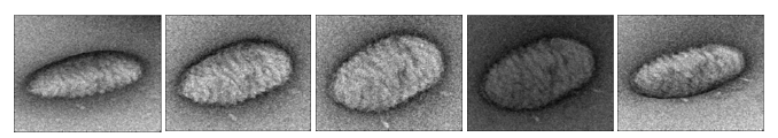

Fig. 5. Orf-parapoxvirus projections respectively according to the angles $-60^{\circ},-40^{\circ},-10^{\circ}, 20^{\circ}$, and $50^{\circ}$.

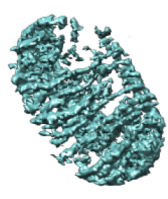

(a)

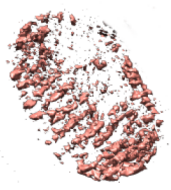

(b)

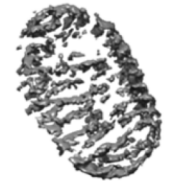

(c)
Fig. 6. Reconstruction of the Orf-parapoxvirus: (a) by our method; (b) reconstruction after alignment by [11]; (c) the reference reconstructed volume.

\section{CONCLUSION}

In this paper, we describe a method to refine an object reconstruction from a set of its tomographic projections and simultaneously correct the errors over all rigid transformations including the used tilt angles. The starting point of the proposed method is an approximation of the object (provided by some reconstruction algorithm) together with a set of given tilt angles used to acquire the projections. Then a cost depending on these angles, the translations (initially there is no translation) and the values of the reconstructed volume, is minimized with a non-linear conjugate gradient. In addition, a multi-scale approach is used to speed up the optimization process.

Our experiments show that the proposed method improves the reconstruction of the object in comparison with a method based on the standard 2D alignment. Further improvements of the reconstruction can still be obtained in the frame of our method. In our future work, we plan to incorporate other parameters such as a priori information about the object to be reconstructed.

Acknowledgement We thank Pr. Patrick SCHULTZ for providing the real data set, the reference reconstructed volume of the Orf-parapoxvirus. 


\section{REFERENCES}

[1] O. Ersen, C. Hirlimann, M. Drillon, J. Werckmann, F. Tihay, C. Pham-Huu, C. Crucifix, and P. Schultz, "3dtem characterization of nanometric objects," Solid State Sciences, vol. 9, no. 12, pp. 1088 - 1098, 2007.

[2] P.A. Penczek, "Chapter one - fundamentals of threedimensional reconstruction from projections," in CryoEM, Part B: 3-D Reconstruction, J.J. Grant, Ed., vol. 482 of Methods in Enzymology, pp. 1-33. Academic Press, 2010.

[3] R. Gordon, R. Bender, and G.T. Herman, "Algebraic Reconstruction Techniques (ART) for three-dimensional electron microscopy and X-ray photography," Journal of Theoretical Biology, vol. 29, no. 3, pp. 471-481, 1970.

[4] P. Gilbert, "Iterative methods for the three-dimensional reconstruction of an object from projections," Journal of Theoretical Biology, vol. 36, no. 1, pp. 105-117, 1972.

[5] R.F. Egerton, P. Li, and M. Malac, "Radiation damage in the $\{$ TEM $\}$ and $\{$ SEM $\}, "$ Micron, vol. 35, no. 6, pp. 399-409, 2004, International Wuhan Symposium on Advanced Electron Microscopy.

[6] S.M. Stagg, G.C. Lander, J. Pulokas, D. Fellmann, A. Cheng, J.D. Quispe, S.P. Mallick, R.M. Avila, B. Carragher, and C.S.Potter, "Automated cryo-em data acquisition and analysis of 284742 particles of groel," Journal of Structural Biology, vol. 155, pp. 470-481, 2006.

[7] C. Colliex, The Electron Microscopy, Presses Universitaires de France, 1998.

[8] J. Frank, Electron tomography: methods for threedimensional visualisation of structures in the cell, Springer, 2006.

[9] S.S. Brandt and U. Ziese, "Automatic TEM image alignment by trifocal geometry," Journal of Microscopy, vol. 222, pp. 1-14, 2006.

[10] C. Sorzano, C. Messaoudi, M. Eibauer, J. Bilbao-Castro, R. Hegerl, S. Nickell, S. Marco, and J. Carazo, "Markerfree image registration of electron tomography tiltseries," BMC Bioinformatics, vol. 10, no. 1, pp. 124, 2009.

[11] J.P.W. Pluim, J.B.A. Maintz, and M.A. Viergever, "Mutual-information-based registration of medical images: a survey," Medical Imaging, IEEE Transactions on, vol. 22, no. 8, pp. 986-1004, Aug 2003.

[12] Q.S. Zheng, J.W. Sedat, and D.A. Agard, "Automated data collection for electron microscopic tomography.,"
Methods in Enzymology, vol. 481, no. 10, pp. 283-315, 2010.

[13] W.O. Saxton, W. Baumeister, and M. Hahn, "Three-dimensional reconstruction of imperfect two-dimensional crystals," Ultramicroscopy, vol. 13, no. 12 , pp. $57-70,1984$.

[14] R. Guckenberger, "Determination of a common origin in the micrographs of tilt series in three-dimensional electron microscopy," Ultramicroscopy, vol. 9, no. 12, pp. $167-173,1982$.

[15] J. Dengler, "A multi-resolution approach to the $3 \mathrm{~d}$ reconstruction from an electron microscope tilt series solving the alignment problem without gold particles," Ultramicroscopy, vol. 30, no. 3, pp. 337 - 348, 1989.

[16] V.-D. Tran, M. Moreaud, É. Thiébaut, L. Denis, and J. M. Becker, "Inverse Problem Approach for the Alignment of Electron Tomographic Series," Oil \& Gas Science and Technology - Revue d'IFP Energies nouvelles, vol. 69, no. 2, pp. 279-291, 2013.

[17] H. Rojbani, É. Baudrier, B. Naegel, L. Mazo, and A. Hamouda, "Angular uncertainty refinement and image reconstruction improvement in cryo-electron tomography," in The 11th International Conference on Computer Vision Theory and Applications, VISAPP'16, Rome, 2016.

[18] P.M. Joseph, "An improved algorithm for reprojecting rays through pixel images," Medical Imaging, IEEE Transactions on, vol. 1, no. 3, pp. 192-196, Nov 1982.

[19] B. De Man and S. Basu, "Distance-driven projection and backprojection in three dimensions," Physics in Medicine and Biology, vol. 49, no. 11, pp. 2463, 2004.

[20] J. Nocedal and S. Wright, Numerical Optimization, Springer Series in Operations Research and Financial Engineering. Springer-Verlag New York, 2 edition, 2006.

[21] Y. H. Dai and Y. Yuan, "A nonlinear conjugate gradient method with a strong global convergence property," SIAM J. on Optimization, vol. 10, no. 1, pp. 177-182, May 1999.

[22] Y. Meyer, "Wavelets and applications," in International Congress of Mathematics, Kyoto, 1990.

[23] M. Van Heel, G.Harauz, E.V. Orlova, R. Schmidt, and M. Schatz, "A new generation of the $\{$ IMAGIC $\}$ image processing system," Journal of Structural Biology, vol. 116, no. 1, pp. $17-24,1996$. 